Patricia Calderón Campos ${ }^{1}$ José G. Vargas-hernández ${ }^{2}$ Jesús Rafael García Serna ${ }^{3}$
Recebido em: 20/04/2015. Aprovado em: 26/05/2015.

1 Profesora del Instituto Tecnológico de Lázaro Cárdenas - Lázaro Cárdenas Michoacán. Cp. 60950 - E-mail: calderon7101@ yahoo.com.mx.

2 M.B.A.; Ph.D. - CUCEA, Universidad de Guadalajara - Periférico Norte 799 Edif. G201-7; Los Belenes; C.P. 45100, Zapopan, Jalisco, México - E-mail: jvargas2006@gmail.com, jgvh0811@yahoo.com, josevargas@cucea.udg. $\mathrm{mx}$.

Profesor de TC del Instituto Tecnológico de Lázaro Cárdenas - Lázaro Cárdenas Michoacán. Cp. 60950 - E-mail: rafael.garciase@otmail.com.

\section{Diseño de una propuesta de mejora de la cultura organizacional en función de las habilidades directivas, en el Instituto Tecnológico de Lázaro Cárdenas*}

\author{
Design of a proposal for improving \\ the organizational culture in terms of \\ management skills, in the Technological \\ Institute of Lazaro Cardenas
}

\section{Resumen}

Día a día se va fortaleciendo más la teoría de que la educación es una parte fundamental para el desarrollo de un país, una educación basada en valores y principios éticos fundamentales que le permita al ser humano un desarrollo armónico que le brinde una mejor calidad de vida. El presente estudio pretende determinar la relación que existe entre las habilidades directivas con la cultura organizacional, ello con el fin de diseñar una propuesta que permita una mejora en la cultura organizacional en el Instituto tecnológico de lázaro Cárdenas, en función de las habilidades directivas, permitiendo establecer estrategias viables y objetivas, que le permitan modificar positivamente su estado actual, partiendo de la hipótesis de que las habilidades directivas de una organización influyen en su cultura organizacional.

Palabras-clave: Cultura organizacional. Habilidades directivas. Instituto Tecnológico.

\begin{abstract}
Every day reinforces the theory that education is the fundamental key to the development of a country, an education based on ethical values and principles that allows humans to the harmonious development and provide a better quality of life. This study aims to determine the relationship between management skills with the culture of the organization, this in order to design a proposal for improving the organizational culture at the Instituto Tecnológico de Lazaro Cárdenas, depending on management skills, allowing viable strategies and objectives, for a positive change in the current situation, assuming the management capacity of an organization, this have influence in your culture organizational.
\end{abstract}

Keywords: Organizational culture. Management skills. Instituto Tecnológico.

JEL: MO, M59 


\section{Introducción}

Uno de los pilares fundamentales y determinantes en el desarrollo social y económico de un estado y un país sin lugar a dudas es la educación, una educación basada en principios y valores éticos que permitan el desarrollo de nuevas generaciones críticas y preparadas académicamente para el desarrollo de los sectores productivos y económicos de los países. La educción es un medio importante que de un desarrollo armónico y sostenido, esta sirve para que un país pueda en un marco de desarrollo y prosperidad.

La cultura organizacional en una Institución educativa, como en cualquier otro tipo de organización se considera fundamental para el crecimiento armónico de esta. Dado a que la cultura es la base fundamental misma que le permite alcanzar niveles de eficiencia y eficacia en el alcance de sus metas, la presente investigación permitió llevar a cabo un análisis detallado de las variables a relacionar la primera cultura organizacional.

Este trabajo de investigación es original dado a que está sustentado con base en experiencias adquiridas a través de la gestión desarrollada como directiva por un periodo de 14 años dentro de los cuales se participó en diferentes en la conducción de diferentes áreas de alto nivel en la institución, desde las jefatura de departamentos hasta llegar a las subdirecciones, aunado a ello se tienen ya 21 años de experiencia docente formando parte de dicha institución

Dicha investigación estará compuesta de un análisis de las principales dimensiones estableciendo por tanto los indicadores de las habilidades directivas, como de la cultura organizacional a través de aplicación de instrumentos (encuestas) aplicadas al personal directivo y docente de la institución, continuando con una revisión de resultados, y concluyendo con la creación de una propuesta de objetiva y viable que permita la mejora en la cultura organizacional del Instituto Tecnológico de Lázaro Cárdenas. Una vez terminada esta fase se continúa con el análisis de la información recompilada a través de las encuestas, cubriendo lo plantado en los objetivos tanto general como específico y comprobando el planteamiento de la hipótesis, hasta llegar finalmente a las conclusiones y recomendaciones que resulten del trabajo, así como proponer nuevas líneas de investigación.

En esta investigación se pone especial atención a seis habilidades que, en opinión de los autores, resumen de alguna forma la relevancia de los comportamientos observables y habituales que justifican el éxito de una persona en su función directiva, éstas son: orientados al conocimiento; liderazgo, comunicación, motivación, toma de decisiones efectivas, negociación y habilidad para trabajar en equipo.

La propuesta de mejora desarrollada impactara de manera directa en generar un cambio en la cultura organizacional del Instituto tecnológico, contribuyendo con esto al establecimiento y mantenimiento de una cultura positiva, solida, efectiva que se manifieste a través del logro de metas institucionales.

\subsection{Objetivos de la investigación}

Los objetivos específicos que a continuación se describen, se desprenden de las dos variables principales que componen el objetivo general de esta investigación (Cultura organizacional y habilidades directivas).

\section{A. Objetivo general}

Diseñar una propuesta de mejora de la cultura organizacional del Instituto Tecnológico de Lázaro Cárdenas, en función de las habilidades directivas.

\section{B. Objetivos específicos}

1) Analizar cuál es el estilo de liderazgo actual en el instituto tecnológico de lázaro Cárdenas y su influencia sobre los valores organizacionales.

2) Determinar la influencia del trabajo en equipo en las actitudes de los trabajadores del instituto tecnológico de lázaro cárdenas.

3) Analizar la influencia que tiene la motivación sobre el comportamiento de los trabajadores del instituto tecnológico de Lázaro Cárdenas.

\section{Antecedentes del problema}

La cultura organizacional es un tema que ha generado un interes desde la década de los ochenta, Sin embargo, este término tiene una amplia gama de perspectivas de estudio que explicamos en este primer apartado como una forma de acercamiento a este concepto.

El concepto de cultura es nuevo en cuanto a su aplicación a la gestión empresarial. Es una nueva óptica que permite a la gerencia comprender y mejorar las organizaciones. Los conceptos que a continuación se plantearán han logrado gran importancia, porque obedecen a 
una necesidad de comprender lo que ocurre en un entorno y explican por qué algunas actividades que se realizan en las organizaciones fallan y otras no.

\section{A. La cultura organizacional}

La cultura organizacional entendida como un factor esencial para el desarrollo de las organizaciones a través del comportamiento de sus entes es imprescindible para el eficiente y efectivo desarrollo de una empresa, el desempeño y las habilidades de los trabajadores sin lugar a dudas es fundamental para que la organización alcance un crecimiento a través el establecimiento de una cultura firme y sostenida de todos los miembros de la organización.

La cultura organizacional es uno de los pilares fundamentales para apoyar a todas aquellas organizaciones que quieren hacerse competitivas. El estudio de la cultura organizacional es un fenómeno relativamente nuevo a nivel mundial; destacando que hasta hace dos décadas eran pocos los autores que se referían este tema. Las primeras obras al respecto difundieron términos como valores, creencias, presunciones básicas, principios; $\mathrm{y}$ otros que inmediatamente se vincularon como constitutivos de un concepto que comenzaba a enraizarse en el campo empresarial.

No existe organización sin una cultura inherente, que la identifique, la distinga y oriente su accionar y modos de hacer, rigiendo sus percepciones y la imagen que sus públicos tengan de ella. Las manifestaciones culturales son a su vez categorías de análisis, a través de las cuales se puede llevar acabo el diagnóstico cultural en una empresa.

De acuerdo con Gordon (1997), la cultura organizacional es un sistema compartido de valores, creencias y hábitos dentro de la organización que interactúan con la estructura formal para producir normas de comportamiento. Desde la prespectiva de Gordon la cultura organizacional se establece en toda empresa o instiucion como un conjunto de acciones interconectadas donde en un todo que hace funcionar a la organización, esta esta soportado por acciones individales que conjuntamente se intercalan para verse como un sistema comun.

Una de las definiciones más referenciadas y posiblemente aceptadas de cultura organizacional es la de Schein (1988, p. 25): Un modelo de presunciones básicas inventadas, descubiertas o desarrolladas por un grupo dado al ir aprendiendo a enfrentarse con sus problemas de adaptación externa e integración interna, que hayan ejercido la suficiente influencia como para ser consideradas válidas y, en consecuencia, ser enseñadas a los nuevos miembros como el modo correcto de percibir, pensar y sentir esos problemas.

Los elementos de la cultura y sus relaciones crean un patròn unico que distigue a una organización tal como la personalidad del individuo es unica. Como ocurre en la clasificacion de las personas que tienen caracteristicas en comun, en el caso de la cultrua organizacional es posible describir varios tipos de cultura Hellregel, Jackson y Slocum (2002 p. 602). Tal como lo explican estos autores, la cultura organizacional estabece una serie de patrones unicos de identidad a la organización, estas igual que en las personas forma parte de sus caracteristicas y valores internos personales, se van construyendo a traves del tiempo.

La cultura organizacional consta de una serie de símbolos, ceremonias y mitos que comunican al personal de la empresa los valores y las creencias más arraigadas dentro de la organización. Estos rituales concretan lo que serían ideas vagas y abstractas, haciendo que cobren vida y significado Ouchi, W. (1981).

Es un modelo de presunciones básicas inventadas, descubiertas o desarrolladas por un grupo dado al ir aprendiendo a enfrentarse con sus problemas de adaptación externa e interna,que hayan ejercido la suficiente influencia como para ser consideradas válidas y, en consecuencia,ser enseñadas a los nuevos miembros como el modo correcto de percibir, pensar y sentir esos problemas Schein, E. (1988).

Para Hofstede, G. (1991) Es un programa mental colectivo que distingue a los miembros de un grupo o categoría de otro. Es compartida por personas cuya vivencia está dentro del mismo ambiente social donde fue aprendida.

\section{B. Las habilidades directivas}

Otro factor a estudiar son las habilidades directivas vistas como las competencias son comportamientos observables y habituales que posibilitan el éxito de una persona en su actividad o función. Las competencias son objetivas en cuanto que tienen consecuencias externas, pero son también subjetivas en cuanto que la percepción de las mismas depende del observador. No ocurre de este modo con los objetivos, que son cuantificables e independientes de la persona que los mide. Por ello, la evaluación 
de las competencias requiere un procedimiento más cuidadoso que la evaluación de los objetivos, ya que hay que tener en cuenta su subjetividad.

Se puede notar que, tradicionalmente, las organizaciones, sean gubernamentales, industriales educativas o médicas, han sido administradas mediante una distribución jerárquica del poder.

La mayor parte de las críticas se centran hacia el ejercicio de la actividad directiva; no cuestionan la legitimidad de los directivos, pero sí la falta de procesos de selección, contratación y evaluación que garanticen la eficacia de su quehacer cotidiano, ya que, sin lugar a dudas, el ejercicio de la dirección se aprende en el desarrollo de la función. Por tal motivo, en lugar de ser un ejercicio sencillo en los primeros meses, es causa de frustración para las personas que conforman la organización. La dirección "es la función ejecutiva de guiar y coordinar para entregar resultados" (TORRES, 2003, p. 33).

De ahí la importancia del desarrollo de las competencias de directivos que está también relacionado con el desarrollo de las competencias de los diferentes actores de las instituciones educativas (subdirectores, directores académicos, directores de investigación, coordinadores de las diferentes áreas, personal docente, administrativo y de apoyo general así como de los alumnos que reciben el servicio educativo) debido a que una dirección eficiente sólo se tendrá en conformación con un equipo honesto y competente que coadyuve para lograr los objetivos y las metas organizacionales. Cuando esto no ocurre, el entorno de la dirección se vuelve complejo.

Un directivo será un líder, o una persona que deberá ser percibida como una figura de autoridad, en una situación, está lo suficientemente seguro de sí mismo y de su relación con los demás de manera tal que experimenta una confianza básica en la capacidad de los demás para pensar por sí mismos, para aprender por sí mismos.

Desde esta perspectiva, y entendiendo que las competencias directivas en escenarios globales constituyen el eje o centro de estas cinco competencias, se desarrollarán en los siguientes apartados el significado y los alcances de cada uno de los atributos gerenciales señalados (o habilidades blandas), entendiendo que cada competencia forma parte y contribuye a desarrollar las competencias directivas necesarias para escenarios globales que se presentan en un modelo conceptual que permitirá comprender la totalidad del conjunto de análisis con un enfoque más sistémico (VILLAREAL, 2008).
No siempre las competencias y/o habilidades han merecido la misma importancia relativa desde la investigación. Hasta hace muy poco se valoraba el conocimiento y dominio de la información como lo más importante, al punto que representó el 75\% de la valoración profesional. Hoy sólo representa el 20 o 25\% (VILLA, 2007), diversificando esta valoración en aspectos tan variados como destreza en la búsqueda de información, trabajo en equipo, liderazgo pedagógico, autoaprendizaje, entre otros.

La competencia sería entonces, la construcción social de aprendizajes significativos y útiles para el desempeño directivo, que se obtiene en gran medida mediante el aprendizaje por experiencia (VILLAREAL JULIAN, 2008).

Mismo que será evaluado dentro del objeto de estudio que es precisamente la cultura organizacional actual del Instituto Tecnológico de Lázaro Cárdenas, los sujetos a investigar son los directivos y docentes que pertenecen a dicha organización, se abordaran dos aspectos importantes, como son la cultura organizacional y las habilidades directivas estableciendo una propuesta para su mejora.

El Instituto Tecnológico de Lázaro Cárdenas (ITALC), nace a partir de la necesidad imperante de contar con una Institución de Educación superior en el municipio en el año 1987, actualmente oferta 8 carreras a nivel licenciatura, cuenta con una matrícula de 2,898 Alumnos y con un total de 153 plantilla de personal integrado por 23 directivos, 117 docentes y 40 trabajadores de asistencia a la educación.

El Instituto se encuentra ubicado como parte de la estructura organizacional dependiente del Sistema Nacional de Institutos Tecnológicos, recientemente denominado Instituto Nacional de México. El Instituto Tecnológico de Lázaro Cárdenas es considerado el número 2 del estado de Michoacán, por sus indicadores, y está localizado en el puerto de Lázaro Cárdenas, el principal puerto del país y uno de los más importantes de América Latina. Sin embargo, a pesar de las características de su ubicación en un polo de desarrollo altamente productivo, este no ha logrado alcanzar los niveles de desarrollo al que podría aspirar dado a las ventajas competitivas que le presenta la región.

Lo anterior se da por una falta de cultura de trabajo presentada en la Institución. No solo no se tiene un trabajo en equipo establecido, sino que también la motivación de sus trabajadores es baja, además de que 
existe una deficiente comunicación formal dentro de las estructuras que conforman las áreas y los departamentos.

En el año 2004, se dio inicio con los trabajos de certificación en la norma ISO 9000, logrando alcanzarla en año 2006, donde se establecía como parte del sistema de calidad una nueva filosofía de trabajo, a través de la gestión de valores y principios de calidad para la mejora, dentro del sistema cada semestre se aplica de manera general a todas las áreas de la institución normativamente una encuesta de ambiente laboral.

Los resultados por lo general establecen una falta de trabajo en equipo, que existe desmotivación en los trabajadores, que los procesos de comunicación y negociación no son los adecuados, estos resultados se dan a conocer y se establecen una serie de cursos de sensibilización que en su gran mayoría solo cubren un indicador necesario para el sistema, sin lograr ninguna mejora en el ambiente organizacional, dado a que solo asisten algunos por indicación bajo una comisión y

Cada 6 años se establece el Programa de Innovación y desarrollo del Instituto que es un documento normativo donde se establece toda una planeación estratégica para el alcance de objetivos y metas anuales, este documento de planeación incluye un apartado en el que se establecen los principios que regirán a la institución, los valores y los indicadores de crecimiento y desarrollo, sin embargo solo se trabaja en el cumplimiento de metas, su alcance a fin de cumplir, sin que esto genere una mejora en la cultura Institucional. No están conectados

Estos esfuerzos de la mejora de la cultura a través de las diferentes estrategias implementadas hasta ahora por la institución, se pueden considerar como esfuerzos aislados y no sistémicos donde se les dé una continuidad y se conozcan las mejoras, a través de un seguimiento y una retroalimentación para conocer su alcance.

Esto es que no existe un cambio planificado, interno y lo más alarmante aun es que a nivel de Tecnológicos de México se trabaja individualmente no se tiene un plan específico de mejora de la cultura a nivel nacional.

Esto debe de ser considerado como un cambio Institucional una mejora de la cultura organizacional se trata de un esfuerzo de cambio conjunto no solo por los directivos si no por todas las personas que integran a la organización, resaltando con esto que esta problemática no es determinada por las personas si no por la falta de una estructura en los por los procesos, así como una nueva filosofía de trabajo.

Por lo antes expuesto se presenta esta investigación con el objetivo de establecer una en una mejora de la cultura organizacional con la responsabilidad y participación de toda la Institución.

La mejora está determinada entonces, en función de las habilidades directivas partiendo de la premisa que los lideres pueden generar los cambios ya que se estos se encuentran posesionados en una zona de control y de influencia importante dentó de la estructura formal de la Institución, y por consiguiente a través de ellos es que se establecen los planes y programas de trabajo y la filosofía con la que se pretende alcanzar las metas, considerando pues que el ejemplo arrastra.

\section{Delimitación del problema}

\section{A. El objeto de estudio}

Por lo antes expuesto por tanto el problema de investigación referencia directamente a la relación que guardan las habilidades directivas en la generación de una cultura organizacional, en este caso específico en una institución de educación superior.

El objeto de estudio es el Instituto Tecnológico de Lázaro Cárdenas, donde es el personal directivo y docente del donde se abordara la relación que guarda la cultura organizacional con las habilidades directivas. El objeto de investigación son 23 directivos y 115 docentes de las 8 diferentes carreras del que se ofrecen en el Instituto Tecnológico de Lázaro Cárdenas., tal como se observa en la Tabla 1.

Tabla 1.

\begin{tabular}{|l|l|l|l|}
\hline Nivel & Directivo/ Docente & No. De funcionarios & total \\
\hline Dirección & Directivo & 1 (uno) & 1 \\
\hline Subdirecciones & Directivo & 3 (tres) & 3 \\
\hline Jefes de departamento & Directivo & 23 (veintitrés) & 23 \\
\hline Carreras de Instituto & Docentes & 118 (ciento dieciocho) & 118 \\
\hline & & Total & 145 \\
\hline \multicolumn{2}{|r}{ Fuente: Elaboración Propia } \\
\end{tabular}

Campo: Educación

Institución: Instituto Tecnológico de Lázaro Cárdenas

Delimitación especial: Personal Directivo y Docentes del Instituto Tecnológico de Lázaro Cárdenas.

Delimitación Temporal: La investigación realizo del 4 de Agosto de 2014 a Octubre del 2015. 


\section{Justificación}

En el Instituto Tecnológico a sus ya casi 27 años se tiene una cultura ya desarrollada y arraigada en sus formas de trabajo, sin embargo esta no ha sido favorable ya que actualmente la institución sigue en nivel de desarrollo y no ha logrado colocarse en el nivel de consolidación que por la situación geográfica en la que se encuentra ya debería haber destacado. El Instituto está situado en una zona privilegiada en un polo de desarrollo importante a nivel nacional e internacional dado a que actualmente el puerto es considerado como el más importante de México y de Latinoamérica por sus características y desarrollo comercial tanto en la importación como la exportación de productos.

Ello debería haber ya colocado a la institución en su más alto nivel dado a que estableciendo convenios con las diferentes empresas tanto nacionales como internacionales, se podría haber pueden desarrollar proyectos de colaboración conjunta hacia el desarrollo científico y tecnológico. Desgraciadamente la institución no tiene una cultura de trabajo que le permita ir creciendo tanto en recursos humanos, tecnológico y por qué no financieros, esto se debe a la falta de una cultura organizacional bien sustentada en principios y valores que generen diversas fuentes de crecimiento y cambio.

Se han hecho intentos como son cursos de sensibilización, se cuenta con la certificación en las normas ISO 9000, ISO 14000, se establecen programas de cursos intersectarles etc., sin embargo no se ha logrado alcanzar ese estatus de trabajo en equipos, se carece de motivación del personal, no se logra una comunicación efectiva, el liderazgo en la mayoría de las áreas es de tipo autocrático.

La finalidad de este trabajo es describir la cultura desde su esencia, analizar la cultura organizacional y empresarial, las habilidades directivas y su impacto con la cultura organizacional, la metodología utilizada es el desarrollo de este proyecto estará basado en un análisis de los temas antes mencionados así como un análisis final donde se determina la importancia de las habilidades directivas para el establecimiento de una cultura organizacional y su transformación a través de estas, estableciendo una conexión directa entre las dos variables, mismas que una vez estudiadas y definidas llevan a una serie de conclusiones que permitan diseñar un propuesta de mejora para la cultura organizacional con base en las habilidades directivas.
La importancia de esta investigación radica principalmente en la solución de problemas de liderazgo, comunicación, motivacional, trabajo en equipo, negociación, toma de decisiones efectivas.

Con esta investigación se intenta contribuir a la solución de la siguiente problemática.

1) La importancia de que mejore el clima laboral a través de esta propuesta incrementaría la productividad, mejoraría la comunicación, el trabajo en equipo, se pretende generar un liderazgo participativo a través del cambio de actitudes y comportamiento.

2) Contar con gente motivada a través de la generación o el establecimiento de valore y cambio de actitudes. Esta propuesta de mejora contribuirá en gran medida a que una institución superior pública pueda ir creciendo conforme a

3) Asimismo, ésta propuesta puede ser transferible y aplicable a otras instituciones que forman parte cualquier institución educativa que forme parte de los Institutos Tecnológicos de México.

4) De igual forma esta propuesta puede ser transferible para aplicarse a otras instituciones con características similares públicas de México.

A continuación se presenta un análisis de los criterios de justificación en la Tabla 2.

Tabla 2.

\begin{tabular}{|c|l|}
\hline Importancia & $\begin{array}{l}\text { Dado a que la cultura organizacional es fundamental para el } \\
\text { desarrollo armónico de esta y genera un crecimiento es importante } \\
\text { conocer }\end{array}$ \\
\hline Aporte práctico & $\begin{array}{l}\text { Con la investigación se contara con una propuesta que permita } \\
\text { desarrollar acciones prácticas que lleven a la mejora de la cultura } \\
\text { organizacional en el objeto de estudio. }\end{array}$ \\
\hline Valor teórico & $\begin{array}{l}\text { La investigación profundizara en el estudio de las teorías relaciona- } \\
\text { das con la cultura organizacional y las habilidades directivas dentro } \\
\text { de instituciones públicas de educación superior. }\end{array}$ \\
\hline Aporte práctico & $\begin{array}{l}\text { La investigación analiza la problemática que se da en las organi- } \\
\text { zaciones por la falta de una cultura y propone una estrategia de } \\
\text { mejora en función de las habilidades directivas. }\end{array}$ \\
\hline Factibilidad & $\begin{array}{l}\text { Es factible dado a que no refiere de recursos económicos, para po- } \\
\text { derse desarrollar, asimismo las encuestas que nos darán el estudio } \\
\text { serán aplicadas en la institución donde se labora actualmente. }\end{array}$ \\
\hline $\begin{array}{l}\text { Impacto y } \\
\text { beneficio }\end{array}$ & $\begin{array}{l}\text { Contar con estrategias de mejora de la cultura que incrementen } \\
\text { los indicadores de crecimiento y desarrollo de las instituciones de } \\
\text { educación superior. }\end{array}$ \\
\hline
\end{tabular}

Fuente: Elaboración propia 


\section{Hipótesis}

\section{Hipótesis de trabajo}

\section{A. Hipótesis general}

\section{$\mathrm{Ho}=\mathrm{Xo} \longrightarrow \mathrm{Yo}$}

Las habilidades directivas de una organización influyen en su cultura organizacional.

\section{B. Hipótesis especificas}

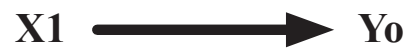

1) Los Estilos de liderazgo influyen sobre los valores organizacionales en el Instituto Tecnológico de lázaro Cárdenas.

\section{$\mathrm{X} 2 \longrightarrow \mathrm{Y}$}

2) Las actitudes de los trabajadores del instituto tecnológico determinan el trabajo en equipo en el Instituto Tecnológico de Lázaro Cárdenas.

\section{$\mathrm{X3} \longrightarrow \mathrm{Y}$}

3) La motivación influencia el comportamiento de los trabajadores del instituto tecnológico de Lázaros Cárdenas.

\section{Marco teórico conceptual}

\subsection{Conceptos de habilidad y de habilidad directiva}

La palabra habilidad por si misma remite a la capacidad, gracia y destreza para ejecutar algo. Guthie Knapp (2007). La palabra habilidad por si misma se remite a la capacidad, gracia y destreza para ejecutar algo las competencias directivas son el nuevo paradigma tanto en la educación como en la práctica laboral que ayudan para alcanzar un mayor desempeño puesto que se ha comprobado su efectividad para fines de capacitación, desarrollo y evaluación profesional.

Cuando se refiriere a las habilidades en plural, mimas que se vinculan a una tarea, implican un entorno, se demuestran en la realización de las tareas con regularidad y eficacia, y, sobre todo, se aprenden. Las habilidades se cultivan y educan. (TORRES, 1998). Estas hacen parte de la capacidad de liderazgo de los grandes gerentes y comprometen un esfuerzo personal y profesional.

Por tanto habilidad es la capacidad del individuo, adquirida por el aprendizaje, capaz de producir resultados previstos con el máximo de certeza, con el mínimo de distendió de tiempo y economía y con el máximo de seguridad. Para efectos de la habilidad directiva, Knapp (2007), señala que la habilidad es la capacidad del individuo adquirida por el aprendizaje, capaz de producir resultados previstos con el máximo de certeza, con el mínimo de distendió de tiempo y economía y con el máximo de seguridad.

Dentro del mundo empresarial se conocen como habilidades directivas todas aquellas cualidades necesarias para manejar la propia vida, así como las relaciones con otros. Estas hacen parte de la capacidad de liderazgo de los grandes gerentes y comprometen un esfuerzo personal y profesional.

Entre las cualidades que a menudo son mencionadas como habilidades directivas suelen encontrarse el liderazgo, la capacidad para trabajar en equipo, las habilidades para motivar a las personas que lo rodean, la capacidad de comunicarse con sus empleados y con el público, la expresión oral, las técnicas de comunicación escrita y las capacidades para relacionarse con la gente. A estos aspectos pueden sumarse la necesidad de contar con una visión crítica, una buena imagen ante el equipo de trabajo y una alta capacidad de innovación.

Las habilidades directivas son aquellas técnicas y habilidades personales que los directivos, independientemente de su área, precisan para desenvolverse profesionalmente y adoptar comportamientos orientados a conseguir la mejor aportación posible de uno mismo y de los demás a los fines de la empresa y gestionar con éxito las relaciones con los demás, la dirección de otras personas o colaboradores y con el entorno. Whetten, D. \& Cameron, K. (2005, p. 76).

Álvarez, C., (1999) define la habilidad "como la dimensión del contenido que muestra el comportamiento del hombre en una rama del saber propio de la cultura de la humanidad. Es, desde el punto de vista psicológico, el sistema de acciones y operaciones dominado por el sujeto que responde a un objetivo."

Por lo anterior se entiende que las habilidades se vinculan a una tarea específica, implican una relación con el entorno, se demuestran en la realización de la tarea con regularidad y eficacia, se aprenden.

Las habilidades directivas son aquellas técnicas y habilidades personales que los directivos, independientemente de su área, precisan para desenvolverse profesionalmente y adoptar comportamientos orientados a conseguir la mejor aportación posible de uno mismo y de los demás a los fines 
de la empresa y gestionar con éxito las relaciones con los demás, la dirección de otras personas o colaboradores y con el entorno. Whetten, D. \& Cameron, K. (2005, p. 76 ).

Las habilidades directivas son fundamentalmente las formas de acción de los líderes, estas están directamente relacionadas con su quehacer diario, mismas que le permiten desenvolverse en la realización de sus funciones, las habilidades directivas son todas aquellas capacidades de acción que tienen los directivos en la conducción de la organización.

Se han realizado diversos estudios sobre la influencia que ejercen las habilidades directivas sobre aspectos fundamentales del crecimiento y desarrollo de las organizaciones, uno de ellos específicamente sobre el clima organizacional, esta investigación desarrollada por Aburto Pineda Hugo, determino que las variables Liderazgo, Comunicación, Motivación, Manejo de conflictos y formación de equipos están directamente ligadas con el clima y la cultura organizacional.

\subsection{Concepto de cultura}

La cultura es el conjunto de valores, costumbres, creencias y prácticas que contribuyen a la forma de vida de un grupo específico (EAGLETON, 2001). Otros autores como Spradley \& McCurdy, definen a la cultura como el conocimiento adquirido que las personas utilizan para interpretar su experiencia y generar comportamientos

Por lo anteriormente expuesto la cultura determina el comportamiento humano a través de las vivencias personales de acuerdo a su nacimiento, los valores con los que se ha crecido, las creencias y todos los conocimientos adquiridos a través del tiempo.

Edward T. Hall propone que la cultura es como un iceberg, ya que la cultura interna engloba a la conducta, creencias y valores de nuestra propia cultura, en si la cultura interna determina o motiva nuestra conducta culturas por enfocarse más en los sentimientos y a las ideas ya que la cultura externa es la que interactúa y tiene conflicto con nuestra propia cultura y puede ser adquirida a través de la observación, educación o a la instrucción, es fácil de aprender y de cambiar por ejemplo las costumbres, comida y lenguaje.

En consecuencia si la cultura interna motiva la conducta y desarrolla los valores, un individuo puede desarrollar un cambio interno a través de nuevas vivencias y la mejora de su educación, su comportamiento cambiara conforme se transforme su entorno.

\subsection{Cultura organizacional}

El concepto de cultura aplicado a la organización se fue gestando desde el aporte de la escuela de las relaciones humanas, cuando a partir de los experimentos desarrollados por Elton Mayo (1972), se empiezan a reconocer los aspectos subjetivos e informales de la realidad organizacional. Mayo (1972), se interesó por indagar acerca de los factores que inciden en el desempeño del trabajador, llegando a la conclusión que el ambiente del grupo al cual pertenece el individuo incide significativamente en la percepción que éste tiene acerca de los aspectos objetivos de la organización.

En lo que se refiere a las organizaciones la cultura es la guía general del comportamiento, creencias compartidas y valores que los miembros tienen en común. (SHEIN, 1992). Por lo expuesto por Shein, la cultura organizacional establece en gran medida un pilar fundamental para el crecimiento y desarrollo de la misma.

Bower (1995), establece que la cultura es un elemento invisible para las personas que pertenecen a las organizaciones, al punto de llegar a convertirse, algunas veces, en la forma de hacer las cosas. Sin embargo, en la raíz de cada cultura están los valores en los que se basa la organización, los cuales pueden ser permanentes y durar muchas décadas o pueden cambiar rápidamente por efecto del tiempo.

Así mismo, Stoner (1996) conceptualiza la cultura organizacional como una serie de entendidos importantes como normas, valores, actitudes y creencias compartidos por los miembros de la organización. Forma actitudes en los empleados y determina la manera en que la organización interactúa con su entorno. Igualmente, Robbins (2004) la designa como un sistema de significados común entre los miembros, que distingue una organización de otras, donde los valores compartidos se conservan y comparten.

Gordon (1997) concibe la cultura organizacional como un sistema compartido de valores, creencias y hábitos dentro de la organización que interactúan con la estructura formal para producir normas de comportamiento.

Mendoza, Hernández y Salazar, mencionan que la cultura organizacional es lo que identifica a una organización, lo que promueve entre sus miembros un sentido de pertenencia, al profesar sus mismos valores, creencias, reglas, procedimientos, normas y lenguaje. 
Asimismo refieren que "La cultura organizacional es aprendida y es una respuesta al entorno, mediante su adaptación a las diferentes situaciones que se presentan, ya que las empresas no tienen la capacidad de controlar el ambiente externo, pero sí de adaptarse, siendo la cultura organizacional un mecanismo que utilizan para ello.

\section{Marco contextual}

El Instituto Tecnológico de Lázaro Cárdenas, Institución de Educación Superior perteneciente al Instituto Tecnológico de México, es coordinado por la Dirección General de Educación Superior Tecnológica "DGEST", ubicado en la Ciudad y Puerto de Lázaro Cárdenas, Michoacán, dispone como oferta educativa de 08 carreras a nivel licenciatura de las cuales 06 son en área de las ingenierías y 02 en el área de las ciencias Económico-Administrativas.

Dichas carreras cuentan con la autorización de los Planes y Programas de Estudio por parte de la Secretaria de Educación Pública y emitidos por la Dirección General de Educación Tecnológica las carreras que actualmente oferta son; Tabla 3.
Tabla 3.

\begin{tabular}{|l|c|}
\hline Programa educativo (carrera) & $\begin{array}{c}\text { No de alumnos por } \\
\text { programa educativo }\end{array}$ \\
\hline Ingeniería Industrial & 467 \\
\hline Ingeniería Electrónica & 337 \\
\hline Ingeniería en Sistemas Computacionales & 294 \\
\hline Licenciatura en Administración (Sistema escolarizado). & 310 \\
\hline Licenciatura en Administración (Sistema Abierto). & 304 \\
\hline Contador Público & 180 \\
\hline Ingeniería Química & 223 \\
\hline Ingeniería Electromecánica & 323 \\
\hline Ingeniería en Gestión Empresarial. & 294 \\
\hline
\end{tabular}

Fuente: Elaboración propia.

Desde el año 1987 nace el Instituto Tecnológico de Lázaro Cárdenas, con el objetivo de formar profesionistas comprometidos con el entorno, participa activamente en el desarrollo social, político y económico, a través de una educación de calidad congruente con el modelo educativo del SNEST. Presenta una opción viable para que la población en general tenga acceso a una formación profesional de calidad.

\section{Propuesta}

\begin{tabular}{|c|c|c|c|c|c|c|}
\hline $\begin{array}{c}\text { Título de la } \\
\text { investigación }\end{array}$ & $\begin{array}{c}\text { Pregunta } \\
\text { general }\end{array}$ & $\begin{array}{l}\text { Preguntas } \\
\text { especificas }\end{array}$ & $\begin{array}{l}\text { Hipótesis } \\
\text { general }\end{array}$ & $\begin{array}{l}\text { Hipótesis } \\
\text { especificas }\end{array}$ & $\begin{array}{l}\text { Objetivo } \\
\text { General }\end{array}$ & $\begin{array}{l}\text { Objetivos } \\
\text { específicos }\end{array}$ \\
\hline \multirow{3}{*}{$\begin{array}{l}\text { Diseño de una pro- } \\
\text { puesta de mejora de la } \\
\text { cultura organizacional } \\
\text { en función de las habi- } \\
\text { lidades directivas, en el } \\
\text { Instituto Tecnológico de } \\
\text { lázaro Cárdenas. }\end{array}$} & \multirow{3}{*}{$\begin{array}{l}\text { ¿Cuáles son las } \\
\text { dimensiones del diseño } \\
\text { de una propuesta de } \\
\text { mejora de la cultura } \\
\text { organizacional del } \\
\text { Instituto Tecnológico } \\
\text { de Lázaro Cárdenas, en } \\
\text { función de las habilida- } \\
\text { des directivas? }\end{array}$} & $\begin{array}{l}\text { 1) ¿Cuál es el estilo de } \\
\text { liderazgo que determi- } \\
\text { na los valores de la cul- } \\
\text { tura organizacional en } \\
\text { el Instituto Tecnológico } \\
\text { de lázaro Cárdenas? }\end{array}$ & \multirow{3}{*}{$\begin{array}{l}\text { Las habilidades } \\
\text { directivas de una orga- } \\
\text { nización influyen en su } \\
\text { cultura organizacional. }\end{array}$} & $\begin{array}{l}\text { 1) Los Estilos de } \\
\text { liderazgo influyen } \\
\text { sobre los valores } \\
\text { organizacionales en el } \\
\text { Instituto Tecnológico de } \\
\text { lázaro Cárdenas y su }\end{array}$ & \multirow{3}{*}{$\begin{array}{l}\text { Diseñar una propuesta } \\
\text { de mejora de la cultura } \\
\text { organizacional del } \\
\text { instituto tecnológico } \\
\text { de lázaro Cárdenas, en } \\
\text { función de las habilida- } \\
\text { des directivas. }\end{array}$} & $\begin{array}{l}\text { 1) Analizar cuál es el } \\
\text { estilo de liderazgo } \\
\text { actual en el instituto } \\
\text { tecnológico de lázaro } \\
\text { Cárdenas y su influen- } \\
\text { cia sobre los valores } \\
\text { organizacionales. }\end{array}$ \\
\hline & & $\begin{array}{l}\text { 2) ¿Cuál es la influencia } \\
\text { de las actitudes que de- } \\
\text { terminan el trabajo en } \\
\text { equipo en el Instituto } \\
\text { Tecnológico de lázaro } \\
\text { Cárdenas?? }\end{array}$ & & $\begin{array}{l}\text { 2) Las actitudes de } \\
\text { los trabajadores del } \\
\text { instituto tecnológico } \\
\text { determinan el trabajo } \\
\text { en equipo. }\end{array}$ & & $\begin{array}{l}\text { 2) Determinar la in- } \\
\text { fluencia del trabajo en } \\
\text { equipo en las actitudes } \\
\text { de los trabajadores del } \\
\text { instituto tecnológico de } \\
\text { lázaro cárdenas. }\end{array}$ \\
\hline & & $\begin{array}{l}\text { 3)¿Cuál es la influencia } \\
\text { que tiene la motivación } \\
\text { sobre el comporta- } \\
\text { miento en la cultura } \\
\text { organizacional en el } \\
\text { instituto tecnológico de } \\
\text { lázaros Cárdenas }\end{array}$ & & $\begin{array}{l}\text { 3) La motivación } \\
\text { influencia el } \\
\text { comportamiento de } \\
\text { los trabajadores del } \\
\text { instituto tecnológico de } \\
\text { Lázaros Cárdenas. }\end{array}$ & & $\begin{array}{l}\text { 3) Analizar la } \\
\text { influencia que tiene } \\
\text { la motivación sobre el } \\
\text { comportamiento de } \\
\text { los trabajadores del } \\
\text { instituto tecnológico de } \\
\text { Lázaros Cárdenas. }\end{array}$ \\
\hline
\end{tabular}




\section{Constructo}
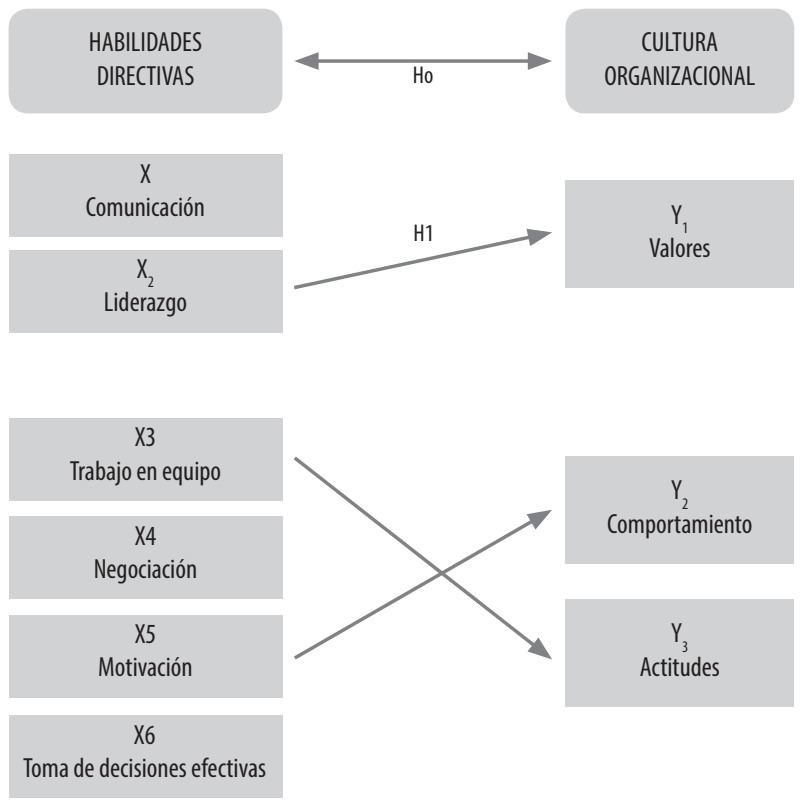

Fuente: Elaboración propia.

\section{Conclusiones}

La educación superior es fundamental para el desarrollo de un país, un estado o una región, dado a que contribuye con en su crecimiento a través de la fuerza de trabajo. Las instituciones de educación deben de ser espacios de conocimiento teórico científico pero además deben de fungir como formadoras integrales de los individuos creando en ellos un sentido de integridad.

Por tanto las instituciones deben de ser entes donde prevalezca una cultura armónica entre sus miembros de forma interna. La cultura organizacional es fundamental es pues un determinante para el logro de las metas y el desarrollo institucional.

La función directiva forma parte importante ya que en ella descansan la responsabilidad y la autoridad que ejercidas con paridad contribuirán al logro de la mejora institucional.

\section{Referencias}

A. Whetten David, S. C. (2011). Desarrollo de habilidades directivas. Mexico: Person.

Cárdenas., I. T. (2007). Programa Institucional de Innovacion y Desarrollo 2006-2012. lazaro Cárdenas michoacan.: Direccion General de Educacion Superior Tecnológica.

Cardona, P. (1999). En busca de las Competencias Directivas. IESE • REVISTA DE ANTIGUOS ALUMNOS, 1,2.

Gordon, J. (1997). Comportamiento organizacional. Mexico: Prentice Hall.

Hellrigel D, J. S. (2002). Administracion un enfoque basado en competencias. Thomson.

Hofsted, G. (1999). Culturas y organizaciones. El software mental. Madrid: Alianza.

Jude A. Timothy, S. P. (Decimotercera edicion). Comportamiento Organizacional. Mèxico: Person.

Madrigal Torres, B. E. (2009). Habilidades directivas. Mexico: Mc Wraw Hill.

Ouchi, W. (1981). Teory Z: How American Bisness can meet the japanes Challeng. Adison-Wesley.

Puga Villareal Julian, M. C. (2008). Competencias directivas en escenarios globales. Estudios Gerenciales.

Villa, A. P. (2007). Aprendizaje basado en competencias: una propuesta para la evaluacion de las competencias genericas. Bilbao: Mensajero. 